\title{
Identification of potential biomarkers of lung adenocarcinoma brain metastases via microarray analysis of cDNA expression profiles
}

\author{
HAIYANG SU ${ }^{1}$, ZHENYANG LIN ${ }^{2}$, WEICHENG PENG ${ }^{2}$ and ZHIQIANG HU ${ }^{1}$ \\ ${ }^{1}$ Department of Neurosurgery, Peking University Ninth School of Clinical Medicine; \\ ${ }^{2}$ Department of Neurosurgery, Beijing Shijitan Hospital, Capital Medical University, Beijing 100038, P.R. China
}

Received February 19, 2018; Accepted September 3, 2018

DOI: $10.3892 / 01.2018 .9829$

\begin{abstract}
Brain metastases originating from lung adenocarcinoma (LAD) occur frequently. The aim of the current study was to assess potential biomarkers for the prognosis of lung adenocarcinoma brain metastasis (LAD-BM) through the analysis of gene expression microarrays. The current study downloaded two gene expression datasets, GSE14108 and GSE10245, from the Gene Expression Omnibus database. From GSE14108 and GSE10245, 19 LAD-BM samples and 40 primary LAD samples were selected for analysis. To identify the differentially expressed genes (DEGs), the current study compared the two sample groups, using the limma R package. Subsequently, pathway enrichment analysis was conducted using the Cluster Profiler R package, and the construction of the protein-protein interaction (PPI) network was executed utilizing the Search Tool for the Retrieval of Interacting Genes database. The microRNA-target network was built using the TargetScore $\mathrm{R}$ package. Then, these networks were established and visualized using Cytoscape software. An array of 463 DEGs was identified in the LAD-BM samples, including 256 upregulated and 207 downregulated genes. Based on functional term enrichment analysis using the Gene Ontology database and signaling pathway enrichment analysis using the Kyoto Encyclopedia of Genes and Genomes database, it was identified that the overlapping DEGs were primarily involved in chemokine-associated signal transduction, which may mediate lung cancer cell metastasis to the brain. Chemokine ligand 2, lysozyme, matrix metalloproteinase-2 (MMP-2), lysyl oxidase (LOX) and granzyme B were identified as potential biomarkers according to a topological analysis of the PPI networks. Two notable nodes, MMP-2 and LOX, appeared in
\end{abstract}

Correspondence to: Professor Zhiqiang $\mathrm{Hu}$, Department of Neurosurgery, Peking University Ninth School of Clinical Medicine, 10 Tieyi Road, Haidian, Beijing 100038, P.R. China

E-mail: neuro07@163.com

Key words: lung adenocarcinoma, brain metastases, microRNA, chemokine signaling pathway the PPI network and were key points in the microRNA-target network, as they were regulated by hsa-let-7d. Many DEGs and microRNAs were regarded as prognostic biomarkers for lung adenocarcinoma metastasis in the current study. These DEGs were primarily associated with chemokine-mediated signaling pathways. In addition, MMP-2 and LOX were predicted to be targets of hsa-let-7d.

\section{Introduction}

Brain metastasis (BM) is a common complication of malignant cancers in adulthood (1) and serves as a major cause of cancer-associated cases of mortality $(2,3)$. Lung cancer, particularly non-small cell lung cancer (NSCLC) (4), is the most common primary cancer that metastasizes to the brain. The percent of patients with NSCLC that suffer from BM is $\sim 10 \%$ at the onset of illness (5), with another $25-40 \%$ demonstrating advancing BM during the disease course (6). Although the precise occurrence is unknown, it is predicted that $35-50 \%$ of all patients with NSCLC are afflicted with BM, based on various studies $(4,7)$. Lung adenocarcinoma (LAD) is a common NSCLC with a frequent incidence of developing brain metastases and accounts for over 50\% of all NSCLC brain metastases (1). Once lung cancer cells have spread to the brain, the patient has a very poor prognosis. The median overall survival of untreated and treated brain metastases patients is 4-11 weeks and 4-15 months, respectively $(8,9)$. Prior investigations have established that there are two mechanisms underlying the involvement of LAD metastasis to the brain, including activation of the Wnt/T-cell factor signal pathway (with homeobox B9 and lymphoid enhancer binding factor 1 expression markedly associated with metastatic ability) and the upregulation of plasminogen activator (PA) inhibitory serpins $(10,11)$. Recently, Singh et al (12) revealed that Sparc/osteonectin, cwcv, and kazal-like domains proteoglycan 1 and twist family bHLH transcription factor 2 are necessary moderators of brain metastasis-initiating cells in LAD and play a key role in the metastatic process in the brain.

MicroRNAs (miRNAs or miRs) are non-coding single stranded nucleotides between 19-24 nucleotides in length (13), which may be important factors in the regulation of tumor invasion and metastasis, including that of LAD, by regulating gene 
expression (14). Previous studies have identified that miR-95, miR-378 and miR-145 may regulate certain key pathways in the process of lung cancer cell metastasis to the brain (15-17). However, there are few reports of miRNAs associated with LAD metastasis.

A previous study has identified differentially expressed genes (DEGs) between primary LAD and metastatic brain tumors by cDNA microarray (18). The results of this previous study suggest that those DEGs, including genes coding for cytoskeletal proteins, cellular antigens and plasma membrane proteins, may serve important roles in cell-cell interactions. However, tumor cell metastasis to the brain is a complicated multi-step process and other important molecules that may be involved, and how they regulate the mechanisms of metastasis, remain largely unknown. Therefore, the current study analyzed the GSE14108 and GSE10245 microarray datasets to identify DEGs in primary LADs and BM. Furthermore, the current study performed enrichment analysis and protein-protein interaction (PPI) network construction. Additionally, the hub genes were combined with the TargetScan database to construct a miRNA-target regulatory network. These comprehensive bioinformatics methods provided an opportunity to identify effective biomarkers for the prognosis of LAD metastasis and to better illuminate the underlying molecular pathogenesis of BM.

\section{Materials and methods}

Data resources. The raw data from the GSE14108 and GSE10245 microarray datasets were downloaded from the Gene Expression Omnibus (GEO) (www.ncbi.nlm.nih. gov/geo/) database. From the datasets, 19 LAD-BM samples (GSE14108) (19) and 40 LAD samples (GSE10245) (20) were used in the current study. The platform used for the detection of these microarray data was the GPL570 Human Genome U133 Plus 2.0 Array (Affymetrix; Thermo Fisher Scientific, Inc., Waltham, MA, USA).

Pre-treatment and DEG analysis. Raw microarray data were pre-processed using the Affy package (version 1.48.0; http://bioconductor.org/packages/release/bioc/html/affy.html); robust multi-array average (RMA) was used for background adjustment and the linear scaling method was applied for normalization. Then, the standardized data were summarized based on the Perfect Match-Mismatch difference model (21). The intensity of gene expression was calculated from the hybridization signal of the probe-set (containing multiple probes). When multiple probes corresponded to the same gene, the average value of probe expression was considered the gene expression value. Using the limma package of $\mathrm{R}$ software (version 3.26.9; http://bioconductor.org/packages/release/bioc/html/limma.html), DEGs were identified by comparing the LAD-BM samples with the LAD samples (22). All DEGs were selected according to the following criteria: $\mid \log 2$ (fold-change) $\mid \geq 1.5$ and a false discovery rate of adjusted $\mathrm{P}<0.001$.

Functional enrichment analysis of DEGs. To identify the potential biological processes that were affected, the Bioconductor package 'Cluster Profiler' of $\mathrm{R}$ software (version 3.2.2; http://www.bioconductor.org/packages/release/bioc/html/clusterProfiler.html) $(23,24)$ was used to classify the enriched Gene Ontology (GO) terms. Information in the Kyoto Encyclopedia of Genes and Genomes (KEGG) (http://www.genome. $\mathrm{jp} / \mathrm{kegg} /$ pathway.html) database was used for the pathway enrichment analysis of DEGs (25). $\mathrm{P}<0.05$ was considered to indicate a statistically significant selection of GO terms and KEGG pathways.

PPI network analysis of the DEGs. The PPI networks of the DEGs were constructed utilizing the Search Tool for the Retrieval of Interacting Genes/Proteins (STRING; string-db. org) database (26) and visualized using Cytoscape software (version 3.6.1; http://www.cytoscape.org/). From 787,896 pairs of human protein interactions containing 16,730 genes, DEG-containing interactions were obtained. STRING (https://string-db.org) utilized a combined score (0-1) (27) to assess reliability. Spearman's correlation coefficient was implemented to assess the edge scores, which evaluated the probability of two co-expressed gene pairs in the current study. Each protein was regarded as a node in the network, and the degree of a node was regarded as the number of interactions with other nodes. Hub genes were nodes with $\geq 50$ degrees.

Establishment of the miRNA-target regulatory network. The TargetScore (version 1.12.0) R package (28) was used to predict biological miRNA-gene interactions based on the TargetScan database (http://www.targetscan.org). The current study combined 'targetscan context+score' with 'probabilities of conserved targeting' of the hub gene to calculate the gene 'targetscore' value, utilizing a variational Bayesian-Gaussian mixture model (28). The miRNAs were identified as miRNA-gene interactions according to the criterion 'targetscore' $>0.4$. The miRNA-gene regulatory network was subsequently visualized using Cytoscape software (version 3.6.1).

\section{Results}

Identification of DEGs in LAD-BM. Overall, the current study identified 463 DEGs (256 upregulated and 207 downregulated) between the LAD-BM and LAD samples. A volcano plot (Fig. 1) demonstrated the distribution of DEGs, and a heat map is presented in Fig. 2.

GO terms and KEGG pathways of DEGs. In total, $65 \mathrm{GO}$ terms and 23 KEGG pathways were enriched in DEGs according to the criteria $\mathrm{P}<0.05$. The 20 most significantly enriched GO terms are presented in Table I. The majority of the enriched GO terms contained 'chemokine-mediated signaling pathway', 'collagen catabolic process' and 'blood vessel morphogenesis'. Certain enriched GO terms also included 'angiogenesis', 'extracellular matrix organization' and 'extracellular structure organization'. The enriched KEGG pathways of the DEGs are listed in Table II. The majority of the enriched KEGG pathways, including 'cytokine-cytokine receptor interaction' and 'chemokine signaling pathway' were directly involved in the process of lung cancer metastasis to the brain.

PPI network of DEGs. Using the STRING database, a PPI network was constructed and is presented in Fig. 3. It included 


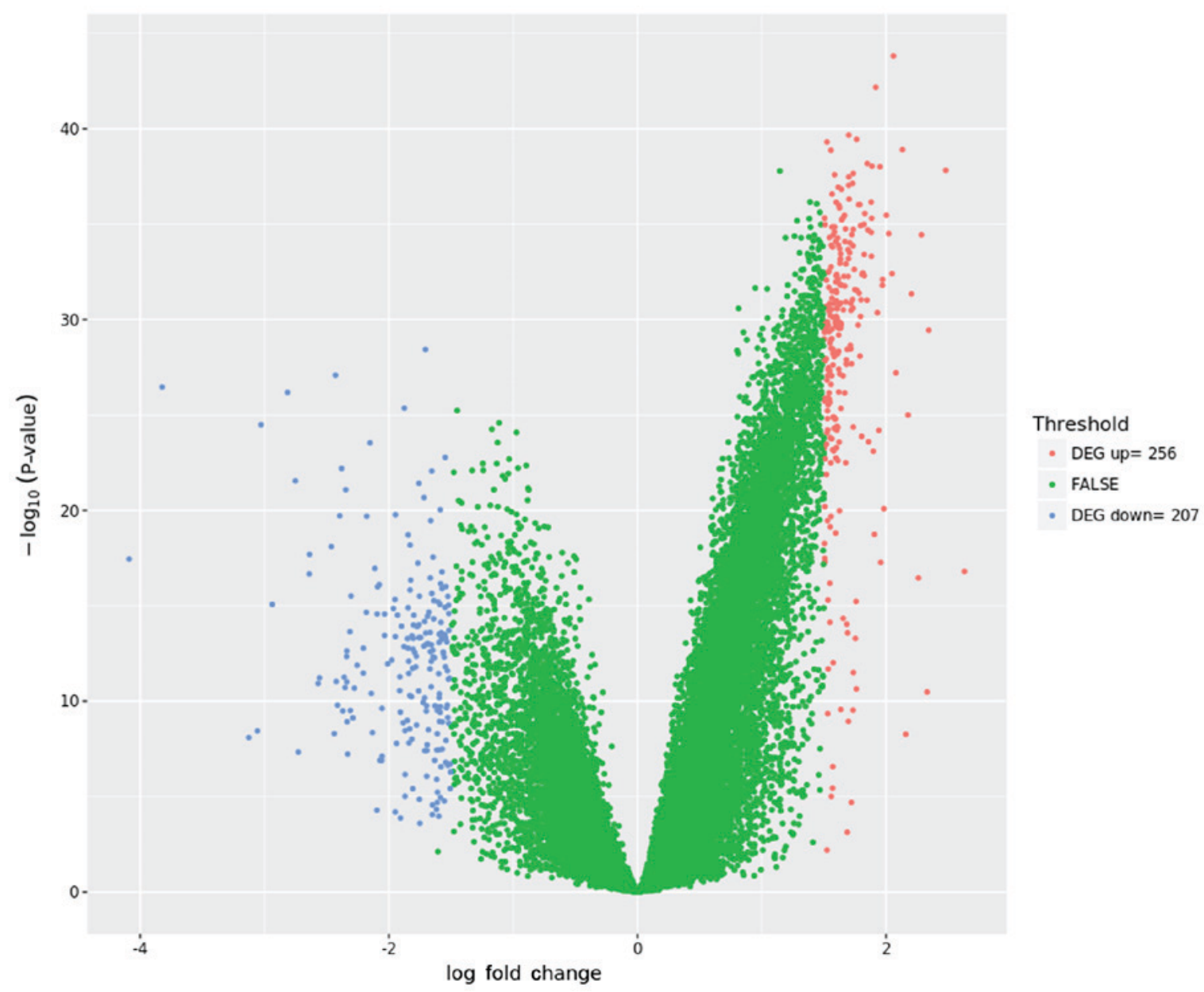

Figure 1. Volcano plot of DEGs. The blue dots represent 207 downregulated DEGs, the red dots represent 256 upregulated DEGs, and the green dots represent non-DEGs. DEG, differentially expressed gene.

942 pairs and 317 nodes. A total of five hub genes were identified, including CCL2 (degree=60), LYZ (degree=60), MMP-2, (degree=58), LOX (degree=53) and GZMB (degree=50).

miRNA-target gene regulatory network. The current study focused on the five hub genes, and further assessed their miRNA-target associations. The miRNA-target regulatory network was based on interactions in the TargetScan database. A miRNA-gene-regulated network was established, including 23 connections and five hub genes, which are presented in Fig. 4. MMP-2 and LOX were significant targets that were identified by and interacted with many miRNAs. MMP-2 was predicted to be the target of the following six miRNAs: hsa-miR-17, hsa-miR-449b, hsa-miR-135a, hsa-miR-530f, hsa-miR-1285 and hsa-let-7d. LOX was predicted to be the target of the following 12 miRNAs: hsa-miR-181a, hsa-miR-155, hsa-miR-26a, hsa-miR-148b, hsa-miR-530f, hsa-miR-182, hsa-miR-200c, hsa-miR-429, hsa-miR-200b, hsa-let-7d, hsa-miR-30b and hsa-miR-27a.

\section{Discussion}

In the current study, the significantly enriched GO terms of DEGs contained 'chemokine-mediated signaling pathway', 'collagen catabolic process' and 'blood vessel morphogenesis'.
As demonstrated by Table II, the enriched KEGG pathways were tumor-associated biological processes, including 'cytokine-cytokine receptor interaction' and 'chemokine signaling pathway'. The overlapping DEGs, including CCL2, CXCL12 and CXCR4, which were mainly enriched in the GO terms and KEGG pathways, were primarily involved in chemokine-associated signal transduction. Numerous studies have previously demonstrated that chemokines serve a pivotal role in cancer invasion, angiogenesis and metastases (29-31). Emerging studies have revealed that the CXCR4/CXCL12 signaling axis is involved in tumor cell migration and angiogenesis, facilitating the formation of BM (32-34). Hartmann et al (35) identified that CXCR4 cooperates with integrins to mediate adhesion and chemo-resistance in small cell lung cancer cells. CXCR4 and CXCL12, which are overexpressed in brain metastases, have been identified to be correlated with brain-specific metastasis and poor prognosis in NSCLC patients (36). Furthermore, the proteolysis of interstitial collagen has been recognized as a contributing factor that participates in tumor cell invasion and metastasis (37). Collagen is one of the chief structural proteins of the extracellular matrix (ECM) and provides a major obstacle impeding cancer cell migration. Thus, it has been speculated that collagen catabolism serves an important role in facilitating the spread and invasion of cancer cells 

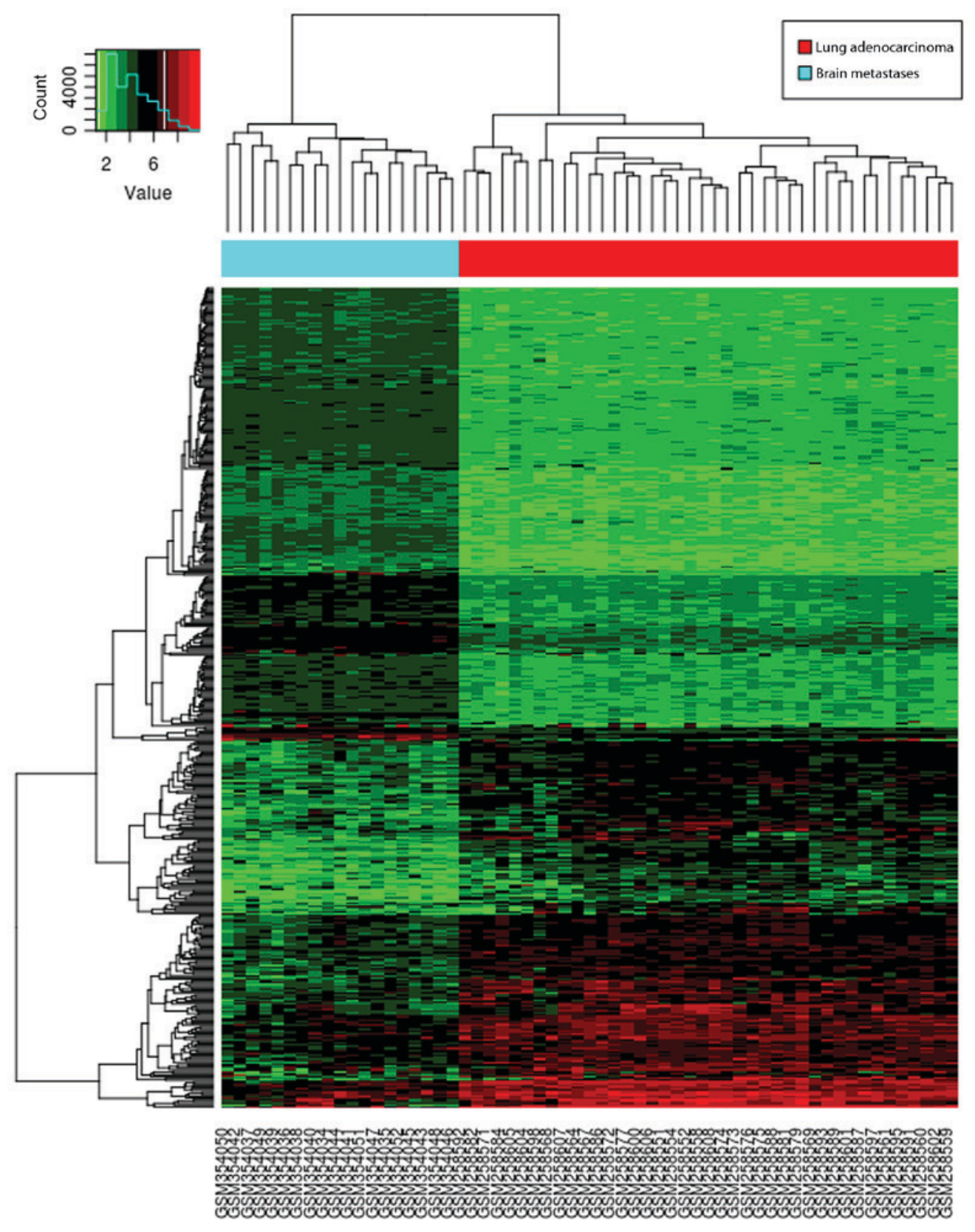

Figure 2. Heat map of gene expression in brain metastases and lung adenocarcinoma samples. The expression level of each gene was normalized and the relative value to the median among 59 samples is demonstrated by color. Red represents high expression and green indicates low expression.

to host organs (38). As demonstrated in Table I, the second most enriched GO term was 'collagen catabolic process'. Indeed, collagen degradation can be observed histologically at the periphery of certain aggressive tumors (39). Thus, the remodeling of the ECM, which is caused by the degradation of collagen, serves an important role in tumor invasion and migration.

The PPI networks based on topology analysis revealed five potential key genes, including CCL2, LYZ, MMP2, LOX and GZMB, which may play important roles in lung cancer BM. CCL2 encodes the chemokine (C-C motif) ligand 2, which interplays with its receptor, $\mathrm{C}-\mathrm{C}$ chemokine receptor type 2 (CCR2), and subsequently promotes tumor progression and metastasis caused by the Snail-induced epithelial-to-mesenchymal transition (EMT) (40). Previous research has reported that the release of CCL2 is induced by activating protease-activated receptor 2 through matrix metalloproteinase-1 expression, which initiates the activation of the thromboxane A2 receptor in LAD cells (41). Furthermore, activation of the thromboxane A2 receptor also increases the expression of CCL2 and then recruits macrophages, thereby stimulating human LAD cell invasion (42). In addition, the CCL2/CCR2 axis cooperates with interleukin-6 to enhance EMT by activating signal transducer and activator of transcription 3-Twist signaling, and then boosts lung cancer progression and metastasis (43).

The matrix metalloprotease (MMP) family includes a series of functionally similar enzyme molecules that degrade protein substrates based on a highly conserved mechanism (44). MMP-2, also known as gelatinase A, has the ability to degrade matrix proteins including gelatin, type IV and type V collagens and elastin (45). MMP-2 is involved in tumor metastasis as it degrades vascular basement membranes (46). Recently, a 
Table I. Top 20 most significantly enriched GO terms for biological processes of differentially expressed genes in brain metastases samples compared with lung adenocarcinoma.

\begin{tabular}{|c|c|c|c|}
\hline GO ID & GO name & $\begin{array}{l}\text { DEG number of } \\
\text { genes involved }\end{array}$ & P-value \\
\hline GO:0070098 & $\begin{array}{l}\text { Chemokine-mediated } \\
\text { Signaling pathway }\end{array}$ & 13 & $3.65 \times 10^{-9}$ \\
\hline GO:0030574 & $\begin{array}{l}\text { Collagen catabolic } \\
\text { process }\end{array}$ & 12 & $2.77 \times 10^{-8}$ \\
\hline GO:0048514 & $\begin{array}{l}\text { Blood vessel } \\
\text { morphogenesis }\end{array}$ & 30 & $2.80 \times 10^{-8}$ \\
\hline GO:0006959 & $\begin{array}{l}\text { Humoral immune } \\
\text { response }\end{array}$ & 18 & $6.75 \times 10^{-8}$ \\
\hline GO:0044236 & $\begin{array}{l}\text { Multicellular organismal } \\
\text { metabolic process }\end{array}$ & 15 & $6.94 \times 10^{-8}$ \\
\hline GO:0044243 & $\begin{array}{l}\text { Multicellular organismal } \\
\text { catabolic process }\end{array}$ & 12 & $7.55 \times 10^{-8}$ \\
\hline GO:0002685 & $\begin{array}{l}\text { Regulation of leukocyte } \\
\text { migration }\end{array}$ & 15 & $1.12 \times 10^{-7}$ \\
\hline GO:0001525 & Angiogenesis & 26 & $1.35 \times 10^{-7}$ \\
\hline GO:0030198 & $\begin{array}{l}\text { Extracellular matrix } \\
\text { organization }\end{array}$ & 25 & $1.45 \times 10^{-7}$ \\
\hline GO:0043062 & $\begin{array}{l}\text { Extracellular structure } \\
\text { organization }\end{array}$ & 25 & $1.52 \times 10^{-7}$ \\
\hline GO:0070661 & Leukocyte proliferation & 20 & $3.00 \times 10^{-7}$ \\
\hline GO:0002688 & $\begin{array}{l}\text { Regulation of leukocyte } \\
\text { chemotaxis }\end{array}$ & 12 & $3.02 \times 10^{-7}$ \\
\hline GO:0030595 & Leukocyte chemotaxis & 16 & $3.15 \times 10^{-7}$ \\
\hline GO:0050920 & Regulation of chemotaxis & 15 & $3.27 \times 10^{-7}$ \\
\hline GO:0060326 & Cell chemotaxis & 18 & $4.57 \times 10^{-7}$ \\
\hline GO:0032963 & $\begin{array}{l}\text { Collagen metabolic } \\
\text { process }\end{array}$ & 13 & $4.88 \times 10^{-7}$ \\
\hline GO:0048247 & Lymphocyte chemotaxis & 9 & $5.20 \times 10^{-7}$ \\
\hline GO:0050795 & Regulation of behavior & 17 & $6.23 \times 10^{-7}$ \\
\hline GO:0044259 & $\begin{array}{l}\text { multicellular organismal } \\
\text { macromolecule metabolic } \\
\text { process }\end{array}$ & 13 & $7.89 \times 10^{-7}$ \\
\hline GO:0002548 & Monocyte chemotaxis & 9 & $1.39 \times 10^{-6}$ \\
\hline
\end{tabular}

GO, gene ontology; ID, identifier; DEG, differentially expressed gene.

study demonstrated that the epigenetic activation of MMP-2 induced by the interaction of megakaryocytic leukemia 1 with histone methyltransferase SET1 can also facilitate the migration and invasion of ovarian tumor cells (47).

The LOX gene encodes lysyloxidase, which is a secretory copper-dependent amine oxidase (48). Previous studies have identified that the overexpression of LOX can also promote tumor cell proliferation, invasion and metastasis in various cancer types (49-51). The downregulation of LOX markedly enhances the expression of E-cadherin and decreases the expression of vimentin (49), increasing the tendency of tumor cells to metastasize. Furthermore, Wilgus et al (52) confirmed that the high expression of LOX is associated with invasion and poor prognosis in patients with LAD.
The most well-known function of lysozyme, encoded by the LYZ gene, is anti-infection (53). Previous research has revealed that the expression of LYZ is associated with an unfavorable prognosis and may be a potential prognostic factor in male breast cancer (54). However, the relationship between LYZ and lung cancer is still not clear.

Additionally, granzyme B is a cytotoxic T-lymphocyteassociated serine esterase. The inhibition of granzyme B and interferon- $\gamma$ activates the transforming growth factor- $\beta / \mathrm{Smad}$ pathway and then inhibits T-cell mediated cancer clearance in vivo, emphasizing the role of the perforin/granzyme pathway in cancer clearance (55). Interestingly, in specific settings, granzyme B and perforin are associated with the regulatory $\mathrm{T}$ cell-mediated inhibition of cancer clearance in vivo (56). 
Table II. The 10 most significantly enriched signaling pathways of differentially expressed genes.

\begin{tabular}{|c|c|c|c|c|}
\hline $\begin{array}{l}\text { KEGG } \\
\text { pathway no. }\end{array}$ & Signaling pathway & DEGs involved & $\begin{array}{l}\text { DEG number of } \\
\text { genes involved }\end{array}$ & P-value \\
\hline hsa04060 & $\begin{array}{l}\text { Cytokine-cytokine } \\
\text { receptor interaction }\end{array}$ & $\begin{array}{l}\text { PDGFRA, CCL2, IL7R, CCL18, } \\
\text { CXCR4, HGF, CXCL12 and others }\end{array}$ & 23 & $1.99 \times 10^{08}$ \\
\hline hsa04145 & Phagosome & $\begin{array}{l}\text { CTSS, HLA-DRA, FCGR2B, } \\
\text { HLA-DPA1, HLA-DQB1, } \\
\text { NOS1, COMP and others }\end{array}$ & 13 & $3.13 \times 10^{05}$ \\
\hline hsa05323 & Rheumatoid arthritis & $\begin{array}{l}\text { IL17A, CCL2, TNFSF13B, } \\
\text { HLA-DRA, HLA-DPA1, CXCL12, } \\
\text { MMP1 and others }\end{array}$ & 10 & $2.55 \times 10^{05}$ \\
\hline hsa05150 & $\begin{array}{l}\text { Staphylococcus aureus } \\
\text { infection }\end{array}$ & $\begin{array}{l}\text { C1R, C1S, FCGR2A, FPR3, } \\
\text { HLA-DRA, FCGR2B, } \\
\text { HLA-DPA1, HLA-DQB1 }\end{array}$ & 8 & $2.69 \times 10^{05}$ \\
\hline hsa04672 & $\begin{array}{l}\text { Intestinal immune } \\
\text { network for IgA production }\end{array}$ & $\begin{array}{l}\text { CXCR4, TNFSF13B, HLA-DRA, } \\
\text { HLA-DPA1, CXCL12, } \\
\text { TNFRSF17, HLA-DQB1 }\end{array}$ & 7 & $7.61 \times 10^{05}$ \\
\hline hsa05320 & $\begin{array}{l}\text { Autoimmune thyroid } \\
\text { disease }\end{array}$ & $\begin{array}{l}\text { IFNA14, IFNA7, HLA-DRA, } \\
\text { HLA-DPA1, HLA-DQB1, GZMB }\end{array}$ & 6 & 0.001016 \\
\hline hsa05164 & Influenza A & $\begin{array}{l}\text { CCL2, SOCS3, CASP1, } \\
\text { HLA-DRA, HLA-DPA1, CCL5, } \\
\text { TNFSF10, HLA-DQB1 and others }\end{array}$ & 11 & 0.001679 \\
\hline hsa04062 & $\begin{array}{l}\text { Chemokine signaling } \\
\text { pathway }\end{array}$ & $\begin{array}{l}\text { CCL2, GNG2, CCL18, } \\
\text { CXCR4, CXCL12, CCL5, CXCL9, } \\
\text { CCL19, CCL8, CXCL13, CXCL11 }\end{array}$ & 11 & 0.002944 \\
\hline hsa04940 & Type I diabetes mellitus & $\begin{array}{l}\text { GAD2, HLA-DRA, HLA-DPA1, } \\
\text { HLA-DQB1, GZMB }\end{array}$ & 5 & 0.002649 \\
\hline hsa05152 & Tuberculosis & $\begin{array}{l}\text { IFNA14, IFNA7, FCGR2A, CTSS, } \\
\text { HLA-DRA, FCGR2B, HLA-DPA1 } \\
\text { and others }\end{array}$ & 10 & 0.005802 \\
\hline
\end{tabular}

KEGG, Kyoto Encyclopedia of Genes and Genomes; DEG, differentially expressed gene; IgA, Immunoglobulin A.

However, Smyth et al (57) discovered that granzyme A and $\mathrm{B}$ are not required for cytotoxic $\mathrm{T}$ cell- and natural killer cell-induced cancer rejection, including spontaneous and experimental cancers. Therefore, more convincing studies are required to confirm the function of granzymes in cancer immune surveillance and rejection.

A miRNA-target regulatory network was constructed, and many potential miRNAs were identified. MMP-2 and LOX were the leading targets identified by and interacting with multiple miRNAs. MMP-2 and LOX were predicted to be targets of hsa-let-7d and hsa-miR-530f. So far, the function of hsa-miR-530f is unclear. Hsa-let-7d reportedly inhibits cancer pathogenesis (58). Furthermore, let-7d may inhibit cancer cell migration, invasion and metastasis, by directly targeting PBX3, COL3A1 and CCL7 $(58,59)$. Meanwhile, a recent study reported that let-7d may suppress proliferation and invasion of trophoblast cells, by targeting MMP-2 (60). However, to the best of our knowledge, the targeting of MMP-2 by hsa-let-7d has not yet been reported in lung cancer. In the current study, MMP-2 and LOX were predicted to be targets of hsa-let-7d, implying that hsa-let-7d may target the two genes during lung cancer metastasis.
The current study also had specific limitations. Certainly, the current results may be more meaningful if matched cases were used to validate the expression of selected genes. Clinically, the most common primary origin of BM is the lung (61), and LAD is usually characterized by the early development of BM, even though certain primary tumors may be observed without symptoms (62). Unfortunately, the current study did not have the opportunity to obtain enough $\mathrm{BM}$ and matched primary tumors to analyze the expression of selected genes in tumor patients. Studies aimed at identifying candidate genes that lead to tumor metastasis are difficult to perform, particularly using human tumor samples. This is due to the unpredictable time point of tumor BM and the difficulty in obtaining tumor tissue samples from patients who have never received chemotherapy or radiotherapy, which is required to ensure that the analysis and interpretation of the results is not confused with drug or radiation-induced changes in gene expression (63). Furthermore, for many years, patients with BM have not generally been considered candidates for surgical intervention, and neurosurgeons have been reluctant to surgically treat these patients due to 


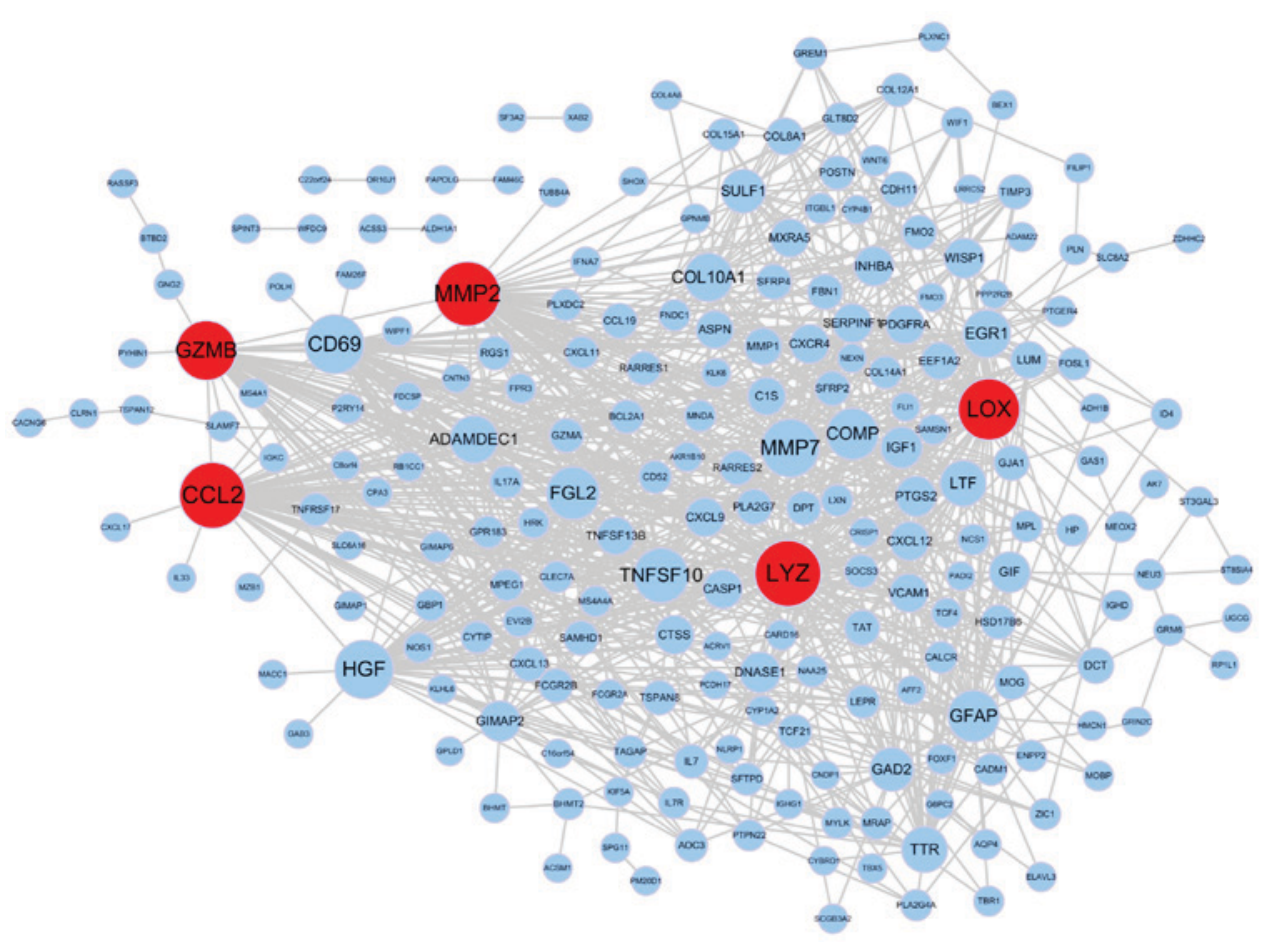

Figure 3. Protein-protein interaction network of differentially expressed genes. Red nodes represent the hub genes ( $\geq 50$ degrees).

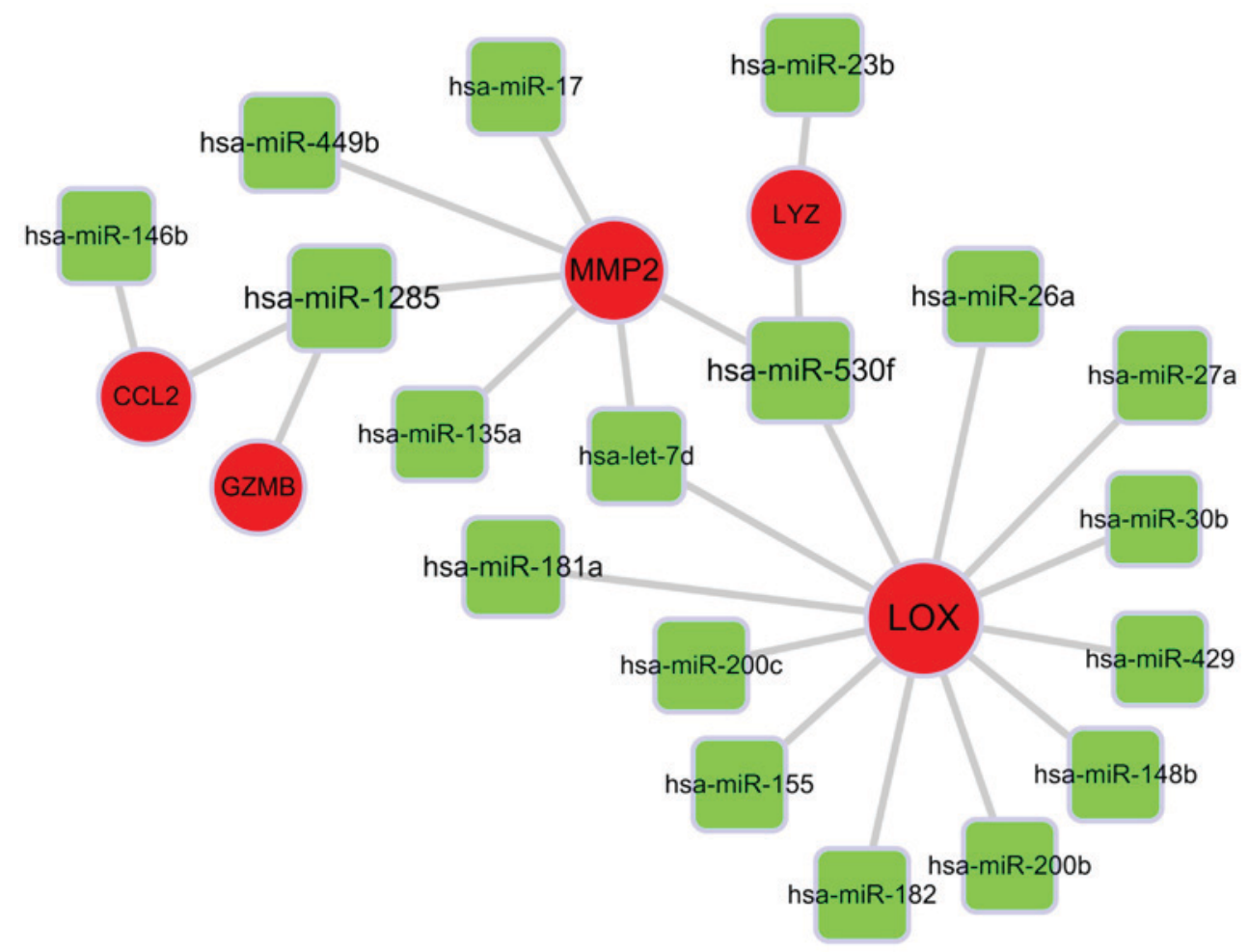

Figure 4. MicroRNA-gene regulatory network of five hub genes in the protein-protein interaction network.

the wide dissemination of cancer and limited survival (64). Prospective or multi-center cooperation may be necessary to collect a well-defined BM group and matched primary tumor cases for further comparative analysis of candidate genes (65). Comprehensive bioinformatics analyses of gene expression profiles based on public databases (e.g. GEO and TCGA) have yielded important insights into potential prognostic biomarkers for tumors (66-68). The current study re-analyzed microarray data from 19 LAD-BM samples and 40 primary LAD samples in the GEO database. All DEGs in LAD-BM compared with the control group were identified via a bioinformatics-based method. Furthermore, the 
current study performed GO term and pathway enrichment analyses, and PPI network construction. The current study also combined the DEG data with information on miRNAs in the TargetScan database to predict miRNA-target interactions. These analyses aided the identification of key genes associated with BM, including CCL2, LYZ, MMP2, LOX and GZMB, and the essential miRNA, hsa-let-7d. Through these comprehensive bioinformatical methods, the current study may contribute to understanding the molecular mechanism underlying BM, facilitating the identification of potential gene targets for the diagnosis and treatment of patients with BM.

In conclusion, many DEGs and miRNAs were regarded as potential biomarkers for the prognosis of LAD metastasis in the present study. These DEGs were mainly associated with chemokine-mediated signaling pathways. In addition, MMP-2 and LOX were predicted to be targets of hsa-let-7d. However, these predictive results require additional experiments to confirm their function.

\section{Acknowledgements}

Not applicable.

\section{Funding}

No funding was received.

\section{Availability of data and materials}

The datasets used and/or analyzed during the present study were obtained from the GEO, KEGG, STRING and TargetScan databases.

\section{Authors' contributions}

HS and $\mathrm{ZH}$ were involved in the conception and design of the research and drafting the manuscript. WP participated in the acquisition of data. HS and ZL performed the analysis and interpretation of data. WP was involved in the statistical analysis. All authors read and approved the final manuscript.

\section{Ethics approval and consent to participate}

Not applicable.

\section{Patient consent for publication}

Not applicable.

\section{Competing interests}

The authors declare that they have no competing interests.

\section{References}

1. Nayak L, Lee EQ and Wen PY: Epidemiology of brain metastases. Curr Oncol Rep 14: 48-54, 2012.

2. Lin NU and Winer EP: Brain metastases: The HER2 paradigm. Clin Cancer Res 13: 1648-1655, 2007.
3. Yau T, Swanton C, Chua S, Sue A, Walsh G, Rostom A, Johnston SR, O'Brien ME and Smith IE: Incidence, pattern and timing of brain metastases among patients with advanced breast cancer treated with trastuzumab. Acta Oncol 45: 196-201, 2006.

4. Hubbs JL, Boyd JA, Hollis D, Chino JP, Saynak M and Kelsey CR: Factors associated with the development of brain metastases. Cancer 116: 5038-5046, 2010.

5. Schuette W: Treatment of brain metastases from lung cancer: Chemotherapy. Lung Cancer 45 (Suppl 2): S253-S257, 2004.

6. Barnholtz-Sloan JS, Sloan AE, Davis FG, Vigneau FD, Lai P and Sawaya RE: Incidence proportions of brain metastases in patients diagnosed (1973 to 2001) in the metropolitan detroit cancer surveillance system. J Clin Oncol 22: 2865-2872, 2004.

7. Shi AA, Digumarthy SR, Temel JS, Halpern EF, Kuester LB and Aquino SL: Does initial staging or tumor histology better identify asymptomatic brain metastases in patients with non-small cell lung cancer? J Thorac Oncol 1: 205-210, 2006.

8. Sundström JT, Minn H, Lertola KK and Nordman E: Prognosis of patients treated for intracranial metastases with whole-brain irradiation. Ann Med 30: 296-299, 1998.

9. Besse B, Le Moulec S, Mazières J, Senellart H, Barlesi F, Chouaid C, Dansin E, Bérard H, Falchero L, Gervais R, et al: Bevacizumab in patients with nonsquamous non-small cell lung cancer and asymptomatic, untreated brain metastases (BRAIN): A nonrandomized, phase II study. Clin Cancer Res 21: 1896-1903, 2015.

10. Nguyen DX, Chiang AC, Zhang XH, Kim JY, Kris MG, Ladanyi M, Gerald WL and Massagué J: WNT/TCF signaling through LEF1 and HOXB9 mediates lung adenocarcinoma metastasis. Cell 138: 51-62, 2009.

11. Valiente M, Obenauf AC, Jin X, Chen Q, Zhang XH, Lee DJ, Chaft JE, Kris MG, Huse JT, Brogi E and Massagué J: Serpins promote cancer cell survival and vascular cooption in brain metastasis. Cell 156: 1002-1016, 2014.

12. Singh M, Venugopal C, Tokar T, Brown KR, McFarlane N, Bakhshinyan D, Vijayakumar T, Manoranjan B, Mahendram S, Vora P, et al: RNAi screen identifies essential regulators of human brain metastasis-initiating cells. Acta Neuropathol 134: 923-940, 2017.

13. Ambros V: The functions of animal microRNAs. Nature 431: 350-355, 2004.

14. Castro D, Moreira M, Gouveia AM, Pozza DH and De Mello RA: MicroRNAs in lung cancer. Oncotarget 8: 81679-81685, 2017.

15. Hwang SJ, Lee HW, Kim HR, Song HJ, Lee DH, Lee H, Shin CH, Joung JG, Kim DH, Joo KM and Kim HH: Overexpression of microRNA-95-3p suppresses brain metastasis of lung adenocarcinoma through downregulation of cyclin D1. Oncotarget 6: 20434-20448, 2015.

16. Chen LT, Xu SD, Xu H, Zhang JF, Ning JF and Wang SF: MicroRNA-378 is associated with non-small cell lung cancer brain metastasis by promoting cell migration, invasion and tumor angiogenesis. Med Oncol 29: 1673-1680, 2012.

17. Zhao C, Xu Y, Zhang Y, Tan W, Xue J, Yang Z, Zhang Y, Lu Y and $\mathrm{Hu} \mathrm{X}$ : Downregulation of miR-145 contributes to lung adenocarcinoma cell growth to form brain metastases. Oncol Rep 30: 2027-2034, 2013.

18. Kikuchi T, Daigo Y, Ishikawa N, Katagiri T, Tsunoda T, Yoshida $\mathrm{S}$ and Nakamura Y: Expression profiles of metastatic brain tumor from lung adenocarcinomas on cDNA microarray. Int J Oncol 28: 799-805, 2006.

19. Kuner R, Muley T, Meister M, Ruschhaupt M, Buness A, Xu EC, Schnabel P, Warth A, Poustka A, Sültmann H and Hoffmann H: Global gene expression analysis reveals specific patterns of cell junctions in non-small cell lung cancer subtypes. Lung Cancer 63: 32-38, 2009.

20. Lüke F, Blazquez R, Yamaci RF, Lu X, Pregler B, Hannus S, Menhart K, Hellwig D, Wester HJ, Kropf S, et al: Isolated metastasis of an EGFR-L858R-mutated NSCLC of the meninges: The potential impact of CXCL12/CXCR4 axis in EGFRmut NSCLC in diagnosis, follow-up and treatment. Oncotarget 9: 18844-18857, 2018.

21. Li C and Wong WH: Model-based analysis of oligonucleotide arrays: Expression index computation and outlier detection. Proc Natl Acad Sci USA 98: 31-36, 2001.

22. Ritchie ME, Phipson B, Wu D, Hu Y, Law CW, Shi W and Smyth GK: Limma powers differential expression analyses for RNA-sequencing and microarray studies. Nucleic Acids Res 43: e47, 2015 .

23. Yu G, Wang LG, Han Y and He QY: ClusterProfiler: An R package for comparing biological themes among gene clusters. OMICS 16: 284-287, 2012. 
24. Yu G, Wang LG, Yan GR and He QY: DOSE: An R/Bioconductor package for disease ontology semantic and enrichment analysis. Bioinformatics 31: 608-609, 2015

25. Ahn T, Lee E, Huh N and Park T: Personalized identification of altered pathways in cancer using accumulated normal tissue data. Bioinformatics 30: 422-429, 2014.

26. Szklarczyk D, Franceschini A, Kuhn M, Simonovic M, Roth A, Minguez P, Doerks T, Stark M, Muller J, Bork P, et al: The STRING database in 2011: Functional interaction networks of proteins, globally integrated and scored. Nucleic Acids Res 39: D561-D568, 2011.

27. Sun Y, Weng Y, Zhang Y, Yan X, Guo L, Wang J, Song X, Yuan Y, Chang FY and Wang CL: Systematic expression profiling analysis mines dys-regulated modules in active tuberculosis based on re-weighted protein-protein interaction network and attract algorithm. Microb Pathog 107: 48-53, 2017.

28. Li Y, Goldenberg A, Wong KC and Zhang Z: A probabilistic approach to explore human miRNA targetome by integrating miRNA-overexpression data and sequence information. Bioinformatics 30: 621-628, 2014.

29. Keeley EC, Mehrad B and Strieter RM: CXC chemokines in cancer angiogenesis and metastases. Adv Cancer Res 106: 91-111, 2010

30. Bachelder RE, Wendt MA and Mercurio AM: Vascular endothelial growth factor promotes breast carcinoma invasion in an autocrine manner by regulating the chemokine receptor CXCR4 Cancer Res 62: 7203-7206, 2002.

31. Akishima-Fukasawa Y, Nakanishi Y, Ino Y, Moriya Y, Kanai Y and Hirohashi S: Prognostic significance of CXCL12 expression in patients with colorectal carcinoma. Am J Clin Pathol 132: 202-10; quiz 307, 2009.

32. Fokas E, Steinbach JP and Rödel C: Biology of brain metastases and novel targeted therapies: Time to translate the research. Biochim Biophys Acta 1835: 61-75, 2013

33. Lee BC, Lee TH, Avraham S and Avraham HK: Involvement of the chemokine receptor CXCR4 and its ligand stromal cell-derived factor lalpha in breast cancer cell migration through human brain microvascular endothelial cells. Mol Cancer Res 2 : 327-338, 2004

34. Hinton CV, Avraham S and Avraham HK: Role of the CXCR4/CXCL12 signaling axis in breast cancer metastasis to the brain. Clin Exp Metastasis 27: 97-105, 2010.

35. Hartmann TN, Burger JA, Glodek A, Fujii N and Burger M: CXCR4 chemokine receptor and integrin signaling co-operate in mediating adhesion and chemoresistance in small cell lung cancer (SCLC) cells. Oncogene 24: 4462-4471, 2005.

36. Salmaggi A, Maderna E, Calatozzolo C, Gaviani P, Canazza A, Milanesi I, Silvani A, DiMeco F, Carbone A and Pollo B: CXCL12, CXCR4 and CXCR7 Expression in brain metastases. Cancer Biol Ther 8: 1608-1614, 2009.

37. Fields GB: Interstitial collagen catabolism. J Biol Chem 288 : 8785-8793, 2013

38. Woolley DE: Collagenolytic mechanisms in tumor cell invasion. Cancer Metastasis Rev 3: 361-372, 1984.

39. Liotta LA, Thorgeirsson UP and Garbisa S: Role of collagenases in tumor cell invasion. Cancer Metastasis Rev 1: 277-288, 1982.

40. Kudo-Saito C, Shirako H, Ohike M, Tsukamoto N and Kawakami Y: CCL2 is critical for immunosuppression to promote cancer metastasis. Clin Exp Metastasis 30: 393-405, 2013.

41. Li X and Tai HH: Thromboxane A 2 receptor-mediated release of matrix metalloproteinase-1 (MMP-1) induces expression of monocyte chemoattractant protein-1 (MCP-1) by activation of protease-activated receptor 2 (PAR2) in A549 human lung adenocarcinoma cells. Mol Carcinog 53: 659-666, 2014.

42. Li X and Tai HH: Activation of thromboxane A2 receptor (TP increases the expression of monocyte chemoattractant protein -1 (MCP-1)/chemokine (C-C motif) ligand 2 (CCL2) and recruits macrophages to promote invasion of lung cancer cells. PLoS One 8: e54073, 2013.

43. Chen W, Gao Q, Han S, Pan F and Fan W: The CCL2/CCR2 axis enhances IL-6-induced epithelial-mesenchymal transition by cooperatively activating STAT3-Twist signaling. Tumor Biol 36: 973-981, 2015

44. Lauer-Fields JL, Juska D and Fields GB: Matrix metalloproteinases and collagen catabolism. Biopolymers 66: 19-32, 2002.

45. Vu TH: Don't mess with the matrix. Nat Genet 28: 202-203, 2001

46. Chakrabarti S and Patel KD: Matrix metalloproteinase-2 (MMP-2) and MMP-9 in pulmonary pathology. Exp Lung Res 31: 599-621, 2005.
47. $\mathrm{Xu} \mathrm{W}, \mathrm{Xu} \mathrm{H}$, Fang $\mathrm{M}, \mathrm{Wu} \mathrm{X}$ and $\mathrm{Xu} \mathrm{Y}$ : MKL1 links epigenetic activation of MMP2 to ovarian cancer cell migration and invasion. Biochem Biophys Res Commun 487: 500-508, 2017.

48. Wang TH, Hsia SM and Shieh TM: Lysyl oxidase and the tumor microenvironment. Int J Mol Sci 18: E62, 2016.

49. Kasashima H, Yashiro M, Kinoshita H, Fukuoka T, Morisaki T, Masuda G, Sakurai K, Kubo N, Ohira M and Hirakawa K: Lysyl oxidase is associated with the epithelial-mesenchymal transition of gastric cancer cells in hypoxia. Gastric Cancer 19: 431-442, 2016.

50. Shih YH, Chang KW, Chen MY, Yu CC, Lin DJ, Hsia SM, Huang HL and Shieh TM: Lysyl oxidase and enhancement of cell proliferation and angiogenesis in oral squamous cell carcinoma. Head Neck 35: 250-256, 2013.

51. Osawa T, Ohga N, Akiyama K, Hida Y, Kitayama K, Kawamoto T, Yamamoto K, Maishi N, Kondoh M, Onodera Y, et al: Lysyl oxidase secreted by tumour endothelial cells promotes angiogenesis and metastasis. Br J Cancer 109: 2237-2247, 2013.

52. Wilgus ML, Borczuk AC, Stoopler M, Ginsburg M, Gorenstein L, Sonett JR and Powell CA: Lysyl oxidase: A lung adenocarcinoma biomarker of invasion and survival. Cancer 117: 2186-2191, 2011.

53. Rubio CA: The natural antimicrobial enzyme lysozyme is up-regulated in gastrointestinal inflammatory conditions. Pathogens 3: 73-92, 2014

54. Serra C, Vizoso F, Alonso L, Rodríguez JC, González LO, Fernández M, Lamelas ML, Sánchez LM, García-Muñiz JL, Baltasar A and Medrano J: Expression and prognostic significance of lysozyme in male breast cancer. Breast Cancer Res 4: R16, 2002.

55. Thomas DA and Massagué J: TGF-beta directly targets cytotoxic $\mathrm{T}$ cell functions during tumor evasion of immune surveillance. Cancer Cell 8: 369-380, 2005.

56. Cao X, Cai SF, Fehniger TA, Song J, Collins LI, Piwnica-Worms DR and Ley TJ: Granzyme B and perforin are important for regulatory $\mathrm{T}$ cell-mediated suppression of tumor clearance. Immunity 27: 635-646, 2007.

57. Smyth MJ, Street SE and Trapani JA: Cutting edge: Granzymes $\mathrm{A}$ and $\mathrm{B}$ are not essential for perforin-mediated tumor rejection. J Immunol 171: 515-518, 2003.

58. Ramberg H, Alshbib A, Berge V, Svindland A and Taskén KA: Regulation of PBX3 expression by androgen and Let-7d in prostate cancer. Mol Cancer 10: 50, 2011.

59. Su B, Zhao W, Shi B, Zhang Z, Yu X, Xie F, Guo Z, Zhang X, Liu J, Shen Q, et al: Let-7d suppresses growth, metastasis, and tumor macrophage infiltration in renal cell carcinoma by targeting COL3A1 and CCL7. Mol Cancer 13: 206, 2014.

60. Dai X and Cai Y: Down-regulation of microRNA let-7d inhibits the proliferation and invasion of trophoblast cells in preeclampsia. J Cell Biochem 119: 1141-1151, 2018

61. Al-Shamy G and Sawaya R: Management of brain metastases: The indispensable role of surgery. J Neurooncol 92: 275-282, 2009.

62. Hoffman PC, Mauer AM and Vokes EE: Lung cancer. Lancet 355: 479-485, 2000

63. Suzuki M and Tarin D: Gene expression profiling of human lymph node metastases and matched primary breast carcinomas: Clinical implications. Mol Oncol 1: 172-180, 2007.

64. Johnson JD and Young B: Demographics of brain metastasis. Neurosurg Clin N Am 7: 337-344, 1996.

65. Preusser M, Berghoff AS, Koller R, Zielinski CC, Hainfellner JA, Liebmann-Reindl S, Popitsch N, Geier CB, Streubel B and Birner P: Spectrum of gene mutations detected by next generation exome sequencing in brain metastases of lung adenocarcinoma. Eur J Cancer 51: 1803-1811, 2015.

66. Zhou S, Liu P, Jiang W and Zhang H: Identification of potential target genes associated with the effect of propranolol on angiosarcoma via microarray analysis. Oncol Lett 13: 4267-4275, 2017.

67. Wang Z, Yang B, Zhang M, Guo W, Wu Z, Wang Y, Jia L, Li S; Cancer Genome Atlas Research Network, Xie W and Yang D: lncRNA epigenetic landscape analysis identifies EPIC1 as an oncogenic lncRNA that interacts with MYC and promotes cell-cycle progression in cancer. Cancer Cell 33: 706-720.e9, 2018.

68. $\mathrm{Li} \mathrm{S}, \mathrm{Li} \mathrm{H}, \mathrm{Xu} \mathrm{Y}$ and $\mathrm{Lv} \mathrm{X}$ : Identification of candidate biomarkers for epithelial ovarian cancer metastasis using microarray data. Oncol Lett 14: 3967-3974, 2017.

This work is licensed under a Creative Commons Attribution-NonCommercial-NoDerivatives 4.0 International (CC BY-NC-ND 4.0) License. 